\title{
EFEITO DAS MUDANÇAS CLIMÁTICAS NA DISPONIBILIDADE HÍDRICA DA BACIA HIDROGRÁFICA DO RIO PARACATU
}

\author{
ELOY L. DE MELLO ${ }^{1}$, FERNANDA A. OLIVEIRA ${ }^{2}$, FERNANDO F. PRUSKI ${ }^{3}$, \\ JULIANA C. FIGUEIREDO ${ }^{4}$
}

\begin{abstract}
RESUMO: O aumento da demanda pelo uso da água na bacia do Rio Paracatu vem ocasionando sérios problemas ambientais e conflitos entre os usuários. Para a gestão mais eficiente dos recursos hídricos, é importante o conhecimento do comportamento hidrológico da bacia hidrográfica, no presente e no futuro, devido às fortes evidências de mudanças climáticas no planeta. $\mathrm{O}$ objetivo deste trabalho foi estimar a tendência de variação da disponibilidade hídrica na bacia hidrográfica do Rio Paracatu, até o final deste século, considerando dois cenários contrastantes de mudanças climáticas, um para altas emissões de $\mathrm{CO}_{2}$ (A2) e outro para baixas (B2). Para atingir esse objetivo, foi realizado o downscaling das precipitações mensais para os anos de 2001 a 2099, simulados pelo modelo de circulação geral do Hadley Centre (HadCM3). As precipitações interpoladas serviram como entrada em modelo do tipo precipitação-vazão, que possibilitou a estimativa das vazões mínimas em 21 estações fluviométricas distribuídas na bacia. Para o cenário A2, verificou-se tendência de aumento na disponibilidade hídrica em todas as estações fluviométricas, variando de 31 a $131 \%$ até 2099. Para o cenário B2, não foi verificada nenhuma tendência significativa.
\end{abstract}

PALAVRAS-CHAVE: downscaling, recursos hídricos, vazões mínimas.

\section{EFFECT OF THE CLIMATE CHANGE ON THE WATER AVAILABILITY IN THE PARACATU RIVER BASIN}

\begin{abstract}
The increase of water use in the Paracatu basin is leading to deep environmental problems and conflicts among the users. For a more efficient water resources management, the knowledge of the basin's hydrologic behavior is important, in the present and in the future, due to the strong climatic changes evidences in the planet. The aim of this work is to estimate the water availability variation trends in the Paracatu river basin, from the beginning until the end of this century, considering two contrasting climate change scenarios, the first one to take account of high $\mathrm{CO}_{2}$ emissions (A2) and the other one for low emissions (B2). To achieve this objective, the monthly precipitation downscaling was accomplished, using data from 2001 to 2099 simulated by the Hadley Centre's global circulation model (HadCM3). The precipitations were used as data input in a precipitation-flow model, which made possible the estimate of the minimum discharges in 21 gauged stations distributed in the basin. For the A2 scenario, an increase trend was verified in the water availability in all of the stations from 2001 to 2099, varying from 31 to $131 \%$. For the B2 scenario no significant trend was verified.
\end{abstract}

KEYWORDS: downscaling, water resources, minimum discharge.

\footnotetext{
${ }^{1}$ Doutorando em Recursos Hídricos e Ambientais, Departamento de Engenharia Agrícola, Universidade Federal de Viçosa, Viçosa MG, eloymello@yahoo.com.br

${ }^{2}$ Mestranda em Recursos Hídricos e Ambientais, Departamento de Engenharia Agrícola, UFV, Viçosa - MG.

${ }^{3}$ Professor Titular, Departamento de Engenharia Agrícola, UFV, Viçosa - MG.

${ }^{4}$ Estudante de Graduação em Engenharia Agrícola e Ambiental, UFV, Viçosa - MG.

Recebido pelo Conselho Editorial em: 22-10-2007
}

Aprovado pelo Conselho Editorial em: 7-10-2008

Eng. Agríc., Jaboticabal, v.28, n.4, p.635-644, out./dez. 2008 


\section{INTRODUÇÃO}

As mudanças climáticas são influenciadas pelas atividades humanas associadas à emissão de gases de efeito estufa e afetam os processos hidrológicos em uma bacia hidrográfica, como a disponibilidade e a qualidade de água. Essas mudanças podem induzir a riscos ambientais, econômicos e sociais, sendo os países mais pobres e em desenvolvimento os mais vulneráveis.

De acordo com o IPCC (2001), o Século XXI deve apresentar maior frequência de eventos extremos de temperatura, precipitação, seca e enchentes, que poderão aumentar os riscos de erosão, deslizamentos de terra, incêndios florestais, riscos à saúde humana e redução do potencial agrícola e da disponibilidade de água para os diversos usos.

De modo geral, os aumentos previstos de temperatura para o Brasil $\left(1\right.$ a $6{ }^{\circ} \mathrm{C}$, dependendo do cenário de emissão de gases), poderiam causar aumento da evaporação à superfície, provocando alterações no balanço hídrico da vegetação natural e das culturas agrícolas (NOBRE, 2001). Esse efeito deve ser mais negativo em regiões onde predominam a agricultura de sequeiro, a não ser que o aumento de temperatura seja acompanhado de aumento e/ou regularização no regime de chuvas.

O efeito projetado das mudanças climáticas no escoamento de água superficial e na recarga de água subterrânea é variável, dependendo da região e do cenário climático considerado (IPCC, 2001), mas relaciona-se, em grande parte, com as mudanças previstas para a precipitação (IPCC, 2001; KROL et al., 2006). É previsto que a magnitude e a frequência de vazões máximas aumentem na maioria das regiões do planeta e que as vazões mínimas sejam menores em muitas regiões.

De acordo com HULME \& SHEARD (1999), é de se esperar que as alterações no regime de precipitação tenham implicações no regime de escoamento dos rios brasileiros, embora não exista tendência bem definida para o aumento ou a redução da vazão. Os autores destacam a forte relação entre a vazão de vários rios da Amazônia e o fenômeno El Niño. Também relatam o aumento de 15\% nas vazões do Rio Paraná desde a década de 1960, consistente com o aumento das precipitações observado nessa década.

A partir de estudo realizado para diversas regiões do mundo, ROSENZWEIG et al. (2004) preveem que o suprimento de água na região Sul do Brasil (Rio Grande do Sul e Santa Catarina) será suficiente para atender à demanda da agricultura e, ainda, promover a expansão das áreas irrigadas.

Na região do semiárido do Nordeste brasileiro, KROL \& BRONSTERT (2007) identificaram tendência significativa de redução nas vazões do Rio Jaguaribe, após 2025, considerando cenário de redução de $50 \%$ da precipitação nas próximas cinco décadas. Em um cenário de redução de $21 \%$ da precipitação, os autores não encontraram tendência significativa de alteração da vazão.

O Rio Paracatu, situado no Médio São Francisco, drena área de aproximadamente $45.600 \mathrm{~km}^{2}$, contribuindo com $40 \%$ da vazão do São Francisco. No entanto, o aumento da demanda pelo uso da água na bacia vem ocasionando sérios impactos ambientais e conflitos entre os usuários.

Segundo RODRIGUEZ (2004), o crescimento econômico que tem sido observado na bacia do Paracatu a partir da década de 1970 vem ocasionando expressivo crescimento no consumo de água, sendo comum, nos meses de maior demanda, consumo total superior ao critério adotado para a outorga em Minas Gerais, que é de $30 \%$ da $\mathrm{Q}_{7,10}$ (vazão mínima com duração de sete dias e período de retorno de 10 anos).

Em uma situação de conflitos pelo uso da água, como se observa na bacia do Paracatu, a gestão participativa torna-se fundamental para o desenvolvimento sustentável da região. Para a gestão eficiente dos recursos hídricos, é importante o conhecimento do comportamento hidrológico da bacia hidrográfica, no presente e no futuro, devido às fortes evidências de mudanças climáticas no planeta.

Cenários de mudanças climáticas são representações plausíveis do futuro, que são consistentes com as tendências de emissão de gases de efeito estufa e outros poluentes, e com o 
entendimento científico atual sobre o efeito desses gases sobre o clima global (IPCC-TGCIA, 1999). Vários cenários podem ser utilizados para identificar a sensibilidade de um país, região, ou local às mudanças climáticas.

Os modelos de circulação global (GCMs) são representações dos processos físicos na atmosfera, no oceano e na superfície da terra, que simulam a resposta do clima global terrestre ao aumento nas concentrações de gases de efeito estufa. Esses modelos descrevem o clima utilizando "grid" tridimensional sobre o planeta, tendo resolução global variando entre 250 e $600 \mathrm{~km}$. Os modelos estão evoluindo sensivelmente a fim de obter resultados confiáveis em resoluções cada vez menores (IPCC, 2007). No entanto, os resultados ainda disponíveis no momento são provenientes de simulações realizadas em "grid" de alta resolução, o que torna difícil a realização de estudos regionais e, principalmente, à escala da bacia hidrográfica, onde as consequências das mudanças climáticas serão realmente sentidas. Nesse caso, é necessário aplicar a técnica de downscaling, que consiste na transferência das informações meteorológicas para escalas menores.

O objetivo deste trabalho foi estimar a tendência de variação da disponibilidade hídrica na bacia hidrográfica do Rio Paracatu, até o final deste século, considerando-se dois cenários de mudanças climáticas.

\section{METODOLOGIA}

\section{Vazões mínimas das séries históricas e precipitação média anual}

Para a realização deste estudo, foram utilizados os dados consistidos correspondentes ao período de 1970 a 2000, de 21 estações fluviométricas (Tabela 1) e 30 estações pluviométricas (Tabela 2) pertencentes à rede hidrometeorológica da Agência Nacional de Águas (ANA). As áreas de drenagem apresentadas na Tabela 1 foram obtidas por meio de mapa digitalizado na escala 1:100.000 correspondente à rede hidrográfica.

TABELA 1. Estações fluviométricas utilizadas no estudo. Flow stations used in this work.

\begin{tabular}{ccccc}
\hline Código & Estação & Latitude & Longitude & Área de Drenagem $\left(\mathrm{km}^{2}\right)$ \\
\hline 42250000 & Fazenda Limoeiro & $1^{\circ} 54^{\prime} 56^{\prime \prime}$ & $47^{\circ} 00^{\prime} 38^{\prime \prime}$ & 490 \\
42251000 & Fazenda Córrego do Ouro & $17^{\circ} 36^{\prime} 48^{\prime \prime}$ & $46^{\circ} 51^{\prime} 31^{\prime \prime}$ & 1.846 \\
42255000 & Fazenda Nolasco & $17^{\circ} 13^{\prime} 48^{\prime \prime}$ & $47^{\circ} 01^{\prime} 20^{\prime \prime}$ & 264 \\
42257000 & Barra do Escurinho & $17^{\circ} 30^{\prime} 45^{\prime \prime}$ & $46^{\circ} 38^{\prime} 46^{\prime \prime}$ & 2.035 \\
42290000 & Ponte da BR-040 (Paracatu) & $17^{\circ} 30^{\prime} 10^{\prime \prime}$ & $46^{\circ} 34^{\prime} 18^{\prime \prime}$ & 7.756 \\
42395000 & Santa Rosa & $17^{\circ} 15^{\prime} 19^{\prime \prime}$ & $46^{\circ} 28^{\prime} 26^{\prime \prime}$ & 12.537 \\
42435000 & Fazenda Barra da Égua & $16^{\circ} 52^{\prime} 28^{\prime \prime}$ & $46^{\circ} 35^{\prime} 12^{\prime \prime}$ & 1.591 \\
42440000 & Fazenda Poções & $17^{\circ} 02^{\prime} 31^{\prime \prime}$ & $46^{\circ} 49^{\prime} 04^{\prime \prime}$ & 545 \\
42460000 & Fazenda Limeira & $16^{\circ} 12^{\prime} 35^{\prime \prime}$ & $47^{\circ} 13^{\prime} 58^{\prime \prime}$ & 3.901 \\
42490000 & Unaí & $16^{\circ} 20^{\prime} 58^{\prime \prime}$ & $46^{\circ} 52^{\prime} 48^{\prime \prime}$ & 5.265 \\
42540000 & Santo Antônio do Boqueirão & $16^{\circ} 31^{\prime} 47^{\prime \prime}$ & $46^{\circ} 43^{\prime} 16^{\prime \prime}$ & 5.844 \\
42545500 & Fazenda o Resfriado & $16^{\circ} 30^{\prime} 10^{\prime \prime}$ & $46^{\circ} 39^{\prime} 46^{\prime \prime}$ & 697 \\
42546000 & Fazenda Santa Cruz & $16^{\circ} 08^{\prime} 06^{\prime \prime}$ & $46^{\circ} 44^{\prime} 52^{\prime \prime}$ & 554 \\
42600000 & Porto dos Poções & $16^{\circ} 50^{\prime} 23^{\prime \prime}$ & $46^{\circ} 21^{\prime} 26^{\prime \prime}$ & 9.419 \\
42690001 & Porto da Extrema & $17^{\circ} 01^{\prime} 49^{\prime \prime}$ & $46^{\circ} 00^{\prime} 49^{\prime \prime}$ & 28.721 \\
42750000 & Caatinga & $17^{\circ} 08^{\prime} 45^{\prime \prime}$ & $45^{\circ} 52^{\prime} 49^{\prime \prime}$ & 31.401 \\
42840000 & Veredas & $18^{\circ} 08^{\prime} 19^{\prime \prime}$ & $45^{\circ} 45^{\prime} 32^{\prime \prime}$ & 185 \\
42850000 & Cachoeira das Almas & $17^{\circ} 21^{\prime} 02^{\prime \prime}$ & $45^{\circ} 31^{\prime} 57^{\prime \prime}$ & 4.370 \\
42860000 & Cachoeira do Paredão & $17^{\circ} 07^{\prime} 16^{\prime \prime}$ & $45^{\circ} 26^{\prime} 08^{\prime \prime}$ & 5.679 \\
42930000 & Porto do Cavalo & $17^{\circ} 01^{\prime} 50^{\prime \prime}$ & $45^{\circ} 32^{\prime} 22^{\prime \prime}$ & 40.869 \\
42980000 & Porto Alegre & $16^{\circ} 46^{\prime} 29^{\prime \prime}$ & $45^{\circ} 22^{\prime} 55^{\prime \prime}$ & 41.868 \\
\hline
\end{tabular}


TABELA 2. Estações pluviométricas utilizadas no estudo. Rain gauge stations used in this work.

\begin{tabular}{|c|c|c|c|c|}
\hline Código & Estação & Latitude & Longitude & Altitude (m) \\
\hline 01546005 & Cabeceiras & $15^{\circ} 48^{\prime} 03^{\prime \prime}$ & $46^{\circ} 55^{\prime} 29^{\prime \prime}$ & 900 \\
\hline 01547002 & Planaltina & $15^{\circ} 27^{\prime} 12^{\prime \prime}$ & $47^{\circ} 36^{\prime} 48^{\prime \prime}$ & 1.000 \\
\hline 01645000 & São Romão & $16^{\circ} 22^{\prime} 18^{\prime \prime}$ & $45^{\circ} 04^{\prime} 58^{\prime \prime}$ & 472 \\
\hline 01645002 & Santo Inácio & $16^{\circ} 16^{\prime} 54^{\prime \prime}$ & $45^{\circ} 24^{\prime} 51^{\prime \prime}$ & 460 \\
\hline 01645003 & Barra do Escuro & $16^{\circ} 16^{\prime} 07^{\prime \prime}$ & $45^{\circ} 14^{\prime} 16^{\prime \prime}$ & 437 \\
\hline 01645009 & Cachoeira da Manteiga & $16^{\circ} 39^{\prime} 25^{\prime \prime}$ & $45^{\circ} 04^{\prime} 51^{\prime \prime}$ & - \\
\hline 01645013 & Fazenda Água Branca & $16^{\circ} 48^{\prime} 26^{\prime \prime}$ & $45^{\circ} 01^{\prime} 49^{\prime \prime}$ & - \\
\hline 01646000 & Porto dos Poções & $16^{\circ} 49^{\prime} 47^{\prime \prime}$ & $46^{\circ} 19^{\prime} 20^{\prime \prime}$ & 540 \\
\hline 01646001 & Unaí & $16^{\circ} 21^{\prime} 05^{\prime \prime}$ & $46^{\circ} 53^{\prime} 23^{\prime \prime}$ & - \\
\hline 01646003 & Santo Antônio do Boqueirão & $16^{\circ} 31^{\prime} 47^{\prime \prime}$ & $46^{\circ} 43^{\prime} 16^{\prime \prime}$ & - \\
\hline 01647001 & Ponte São Bartolomeu & $16^{\circ} 32^{\prime} 16^{\prime \prime}$ & $47^{\circ} 48^{\prime} 02^{\prime \prime}$ & 790 \\
\hline 01647002 & Cristalina & $16^{\circ} 45^{\prime} 23^{\prime \prime}$ & $47^{\circ} 36^{\prime} 22^{\prime \prime}$ & 1.239 \\
\hline 01744006 & Pirapora-Barreiro & $17^{\circ} 21^{\prime} 50^{\prime \prime}$ & $44^{\circ} 56^{\prime} 54^{\prime \prime}$ & 471 \\
\hline 01745000 & Caatinga & $17^{\circ} 08^{\prime} 45^{\prime \prime}$ & $45^{\circ} 52^{\prime} 49^{\prime \prime}$ & 502 \\
\hline 01745001 & Cachoeira do Paredão & $17^{\circ} 06^{\prime} 40^{\prime \prime}$ & $45^{\circ} 26^{\prime} 16^{\prime \prime}$ & 520 \\
\hline 01745007 & Porto do Cavalo & $17^{\circ} 01^{\prime} 37^{\prime \prime}$ & $45^{\circ} 32^{\prime} 26^{\prime \prime}$ & 473 \\
\hline 01746001 & Porto da Extrema & $17^{\circ} 01^{\prime} 51^{\prime \prime}$ & $46^{\circ} 00^{\prime} 49^{\prime \prime}$ & 510 \\
\hline 01746002 & Santa Rosa & $17^{\circ} 15^{\prime} 19^{\prime \prime}$ & $45^{\circ} 28^{\prime} 26^{\prime \prime}$ & 490 \\
\hline 01746006 & Ponte da BR-040 (Prata) & $17^{\circ} 39^{\prime} 49^{\prime \prime}$ & $46^{\circ} 21^{\prime} 18^{\prime \prime}$ & - \\
\hline 01746007 & Ponte da BR-040 (Paracatu) & $17^{\circ} 30^{\prime} 10^{\prime \prime}$ & $46^{\circ} 34^{\prime} 18^{\prime \prime}$ & - \\
\hline 01746008 & Paracatu & $17^{\circ} 13^{\prime} 00^{\prime \prime}$ & $46^{\circ} 52^{\prime} 00^{\prime \prime}$ & - \\
\hline 01747001 & Campo Alegre de Goiás & $17^{\circ} 30^{\prime} 15^{\prime \prime}$ & $47^{\circ} 33^{\prime} 20^{\prime \prime}$ & 800 \\
\hline 01747005 & Guarda Mor & $17^{\circ} 46^{\prime} 21^{\prime \prime}$ & $47^{\circ} 05^{\prime} 55^{\prime \prime}$ & - \\
\hline 01845013 & São Gonçalo do Abaeté & $18^{\circ} 20^{\prime} 37^{\prime \prime}$ & $45^{\circ} 50^{\prime} 12^{\prime \prime}$ & 836 \\
\hline 01846003 & Major Porto & $18^{\circ} 42^{\prime} 25^{\prime \prime}$ & $46^{\circ} 02^{\prime} 13^{\prime \prime}$ & 672 \\
\hline 01846005 & Presidente Olegário & $18^{\circ} 24^{\prime} 45^{\prime \prime}$ & $46^{\circ} 25^{\prime} 20^{\prime \prime}$ & - \\
\hline 01846016 & Ponte Firme & $18^{\circ} 02^{\prime} 02^{\prime \prime}$ & $46^{\circ} 25^{\prime} 10^{\prime \prime}$ & - \\
\hline 01846017 & Leal dos Patos & $18^{\circ} 38^{\prime} 28^{\prime \prime}$ & $46^{\circ} 20^{\prime} 04^{\prime \prime}$ & - \\
\hline 01847003 & Abadia dos Dourados & $18^{\circ} 29^{\prime} 28^{\prime \prime}$ & $47^{\circ} 24^{\prime} 23^{\prime \prime}$ & 784 \\
\hline 01847040 & Fazenda São Domingos & $18^{\circ} 06^{\prime} 11^{\prime \prime}$ & $47^{\circ} 41^{\prime} 41^{\prime \prime}$ & - \\
\hline
\end{tabular}

Para cada estação fluviométrica, foi determinada a $\mathrm{Q}_{7,10}$ (vazão mínima com duração de sete dias e período de retorno de 10 anos), a partir do ajuste de distribuições de probabilidade às $\mathrm{Q}_{7}$ anuais. Foi selecionada a distribuição que apresentou significância a $20 \%$ pelo teste de Kolmogorov-Smirnov, associada ao menor coeficiente de variação.

O cálculo da precipitação média anual sobre cada área de drenagem foi feito pelo método do Polígono de Thiessen.

\section{Modelo para estimativa de vazões mínimas a partir de dados pluviométricos}

Para a caracterização da curva de recessão do escoamento subterrâneo, foi utilizada a eq.(1):

$$
\mathrm{Q}=\mathrm{Q}_{0} \mathrm{e}^{-\alpha \mathrm{t}}
$$

em que,

$\mathrm{Q}$ - vazão subterrânea num instante $\mathrm{t}, \mathrm{m}^{3} \mathrm{~s}^{-1}$;

$\mathrm{Q}_{0}$ - vazão subterrânea correspondente ao início do período de recessão do escoamento subterrâneo, $\mathrm{m}^{3} \mathrm{~s}^{-1}$;

$\alpha$ - coeficiente de recessão, $\mathrm{d}^{-1}$, e

$\mathrm{t}$ - tempo contado a partir do início do período de recessão, $\mathrm{d}$.

Com base no estudo desenvolvido por NOVAES (2005), os dias primeiro de junho e 30 de setembro foram escolhidos como sendo o início e o final do período de recessão do escoamento subterrâneo, respectivamente. Em seguida, foi ajustado um modelo de regressão linear para cada 
uma das 21 estações fluviométricas, visando à representação das $\mathrm{Q}_{0}$ como função da precipitação total acumulada nos cinco meses anteriores ao início do período de recessão do escoamento subterrâneo, ou seja, de janeiro a maio [eq.(2)].

$$
\mathrm{Q}_{0}=\mathrm{a}+\mathrm{bPT}_{5}
$$

em que,

a e b - parâmetros de ajuste da equação, e

$\mathrm{PT}_{5}$ - precipitação total acumulada nos cinco meses anteriores ao início do período de recessão do escoamento subterrâneo, $\mathrm{mm}$.

Os valores dos coeficientes de recessão $(\alpha)$ foram obtidos de NOVAES (2005), que ajustou a eq.(1) aos dados observados das mesmas séries históricas das 21 estações fluviométricas (Tabela 3).

TABELA 3. Valores dos coeficientes de recessão (NOVAES, 2005). Recession coeficient values (NOVAES, 2005).

\begin{tabular}{ccc}
\hline Código & Estação & Coeficiente de Recessão $-\alpha\left(\mathrm{d}^{-1}\right)$ \\
\hline 42250000 & Fazenda Limoeiro & 0,0058 \\
42251000 & Fazenda Córrego do Ouro & 0,0067 \\
42255000 & Fazenda Nolasco & 0,0059 \\
42257000 & Barra do Escurinho & 0,0072 \\
42290000 & Ponte da BR-040 (Paracatu) & 0,0064 \\
42395000 & Santa Rosa & 0,0057 \\
42435000 & Fazenda Barra da Égua & 0,0066 \\
42440000 & Fazenda Poções & 0,0056 \\
42460000 & Fazenda Limeira & 0,0063 \\
42490000 & Unaí & 0,0065 \\
42540000 & Santo Antônio do Boqueirão & 0,0060 \\
42545500 & Fazenda o Resfriado & 0,0046 \\
42546000 & Fazenda Santa Cruz & 0,0029 \\
42600000 & Porto dos Poções & 0,0057 \\
42690001 & Porto da Extrema & 0,0057 \\
42750000 & Caatinga & 0,0059 \\
42840000 & Veredas & 0,0032 \\
42850000 & Cachoeira das Almas & 0,0065 \\
42860000 & Cachoeira do Paredão & 0,0064 \\
42930000 & Porto do Cavalo & 0,0059 \\
42980000 & Porto Alegre & 0,0054 \\
\hline & & 0,0058 \\
\hline
\end{tabular}

\section{Modelo de previsão de mudanças climáticas e downscaling}

Foi realizado o downscaling estatístico das precipitações mensais para os anos de 2001 a 2099, simulados pelo modelo de circulação global HadCM3, para os cenários de alta e de baixa emissão de gases de efeito estufa, A2 e B2, respectivamente.

O cenário A2 caracteriza um mundo onde prevalece o fortalecimento das identidades culturais regionais, com ênfase aos valores familiares e tradições locais, alto crescimento populacional e menor preocupação com o rápido desenvolvimento econômico. O cenário B2 descreve um mundo onde as soluções para os problemas econômicos, sociais e ambientais são desenvolvidas por iniciativas locais e comunitárias, ao invés de buscar soluções globais. É um mundo heterogêneo, com mudanças tecnológicas mais lentas e diversificadas (IPCC, 2001).

O modelo HadCM3 apresenta resolução horizontal de 2,5 de latitude x 3,75 de longitude, o que produz um "grid" global de 96 x 73 células. A partir de análise prévia com auxílio de um SIG, foram determinadas as células que estavam sobre a bacia hidrográfica do Paracatu (Figura 1). Depois disso, para cada uma das 21 estações fluviométricas, foi determinada a proporção da área de drenagem que está sob cada célula. Para a realização do downscaling, foi utilizada a eq.(3): 


$$
\mathrm{P}_{\mathrm{ma}}=\mathrm{FC}\left[\left(\mathrm{P}_{\mathrm{mf}, \mathrm{c} 1} \mathrm{~A}_{\mathrm{c} 1}\right)+\left(\mathrm{P}_{\mathrm{mf}, \mathrm{c} 2} \mathrm{~A}_{\mathrm{c} 2}\right)+\left(\mathrm{P}_{\mathrm{mf}, \mathrm{c} 3} \mathrm{~A}_{\mathrm{c} 3}\right)+\left(\mathrm{P}_{\mathrm{mf}, \mathrm{c} 4} \mathrm{~A}_{\mathrm{c} 4}\right)\right]
$$

em que,

$\mathrm{P}_{\mathrm{ma}}$ - precipitação estimada para o mês "m" do ano "a", sobre a área de drenagem considerada, $\mathrm{mm}$;

$\mathrm{P}_{\mathrm{mf}, \mathrm{c} 1 \ldots} \mathrm{P}_{\mathrm{mf}, \mathrm{c} 4}$ - precipitação estimada para o mês "m" do ano "a", na célula $1 ; 2 ; 3$ e 4, respectivamente, previsto pelo $\mathrm{HadCM} 3, \mathrm{~mm}$;

$\mathrm{A}_{\mathrm{c} 1 \ldots} \mathrm{A}_{\mathrm{c} 4}$ - proporção da área de drenagem da estação sob a célula $1 ; 2 ; 3$ e 4, respectivamente, adimensional.

FC é um fator de conversão calculado pela eq.(4):

$\mathrm{FC}=\frac{\mathrm{P}_{\mathrm{mm}, \mathrm{Ad}}}{\left(\mathrm{P}_{\mathrm{mm}, \mathrm{c} 1} \mathrm{~A}_{\mathrm{c} 1}\right)+\left(\mathrm{P}_{\mathrm{mm}, \mathrm{c} 2} \mathrm{~A}_{\mathrm{c} 2}\right)+\left(\mathrm{P}_{\mathrm{mm}, \mathrm{c} 3} \mathrm{~A}_{\mathrm{c} 3}\right)+\left(\mathrm{P}_{\mathrm{mm}, \mathrm{c} 4} \mathrm{~A}_{\mathrm{c} 4}\right)}$

em que,

$\mathrm{P}_{\mathrm{mm}, \mathrm{Ad}}$ - precipitação total mensal na área de drenagem, com base na série histórica de $1970 \mathrm{a}$ 2000, mm;

$\mathrm{P}_{\mathrm{mm}, \mathrm{c} 1} \ldots \mathrm{P}_{\mathrm{mm}, \mathrm{c} 4}$ - precipitação total mensal nas células 1; 2; 3 e 4, respectivamente, com base na série histórica de 1970 a 2000, mm.

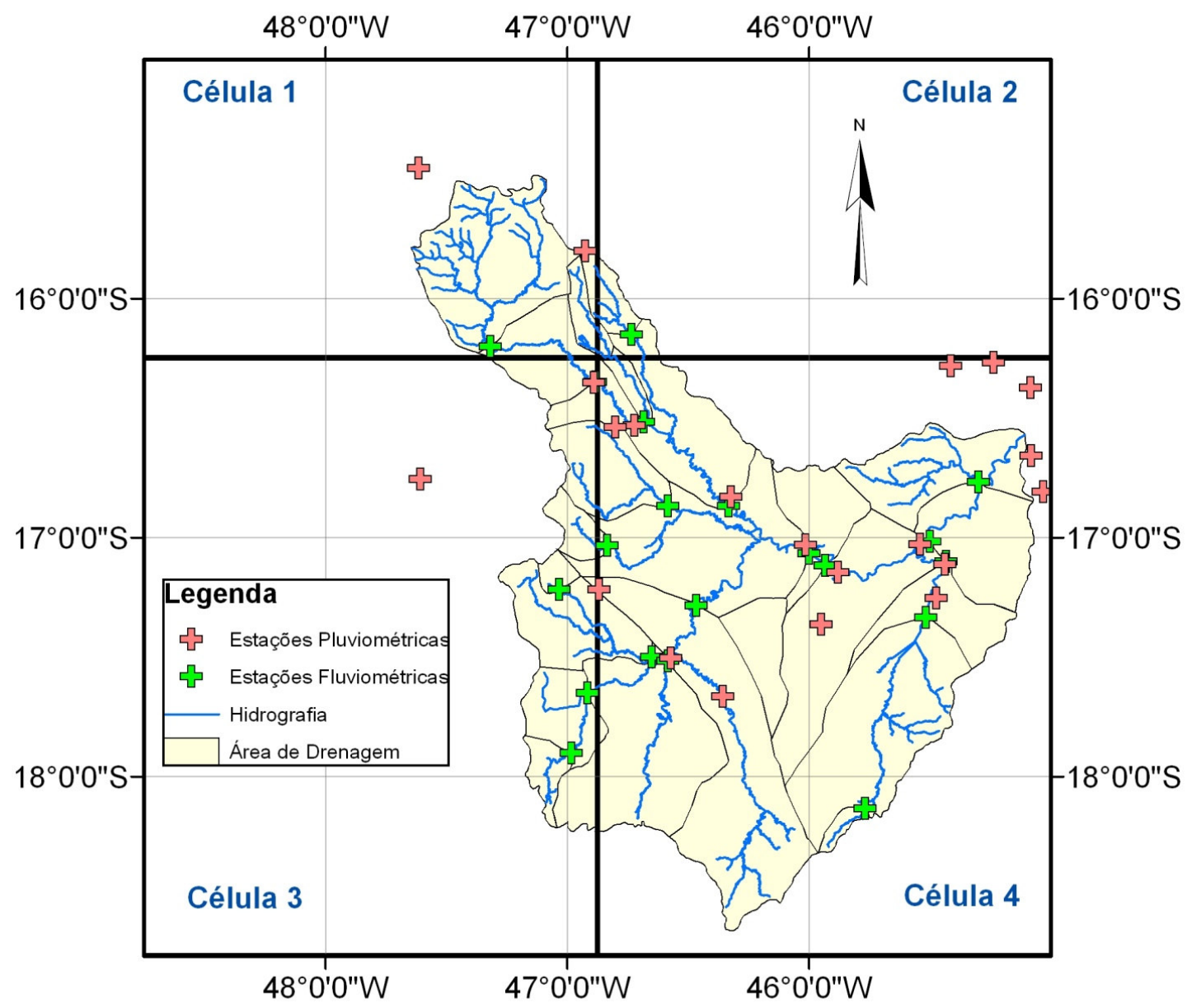

FIGURA 1. Células do modelo de circulação global sobre a bacia do Paracatu, estações pluviométricas e fluviométricas e áreas de drenagem. Global circulation model pixels over the Paracatu basin, rain and flow gauged stations and sub-basins. 
A $\mathrm{PT}_{5}$, para cada ano de 2001 a 2099, foi calculada pela soma das precipitações mensais de janeiro até maio de cada ano, que foram obtidas por meio do downscaling.

\section{Obtenção das vazões mínimas para as próximas décadas}

Obtidos os valores de $\mathrm{PT}_{5}$, os mesmos foram aplicados na eq.(2), que foi substituída, em seguida, na eq.(1). Dessa maneira, foi possível estimar as curvas de recessão do escoamento para cada ano, de 2001 a 2099. Conhecendo-se as curvas de recessão, foram calculados os valores de $\mathrm{Q}_{7}$ para cada ano, e os valores de $\mathrm{Q}_{7,10}$ com base em cada década. Para o período de 1970 a 2000, foi calculada a $\mathrm{Q}_{7,10}$ com base na série histórica de cada estação.

Para facilitar as comparações, os valores de $\mathrm{Q}_{7,10}$ foram divididos pelas respectivas áreas de drenagem, obtendo-se, assim, as $\mathrm{Q}_{7,10}$ específicas $\left(\mathrm{q}_{7,10}\right)$. Por fim, a tendência das $\mathrm{q}_{7,10}$ foi analisada por regressão linear, tendo o tempo como variável independente.

\section{RESULTADOS E DISCUSSÃO}

Na Figura 2, é apresentada a $\mathrm{PT}_{5}$ sobre a bacia do Paracatu, de 1970 a 2000 (dados observados), e de 2001 a 2099, considerando o modelo de previsão de mudanças climáticas (HadCM3) e os dois cenários de emissão de gases utilizados neste trabalho.

A análise desses resultados permitiu observar que no período de 1970 a 2000, a $\mathrm{PT}_{5}$ média foi de $876 \mathrm{~mm}$. Considerando as previsões do modelo de circulação geral, espera-se aumento na $\mathrm{PT}_{5}$ média para o período de 2001 até 2059 nos dois cenários, sendo esperado aproximadamente 1.002 mm para o cenário A2, e $1.005 \mathrm{~mm}$ para o cenário B2, embora fique evidenciada na Figura 2 a ocorrência de variações sazonais.

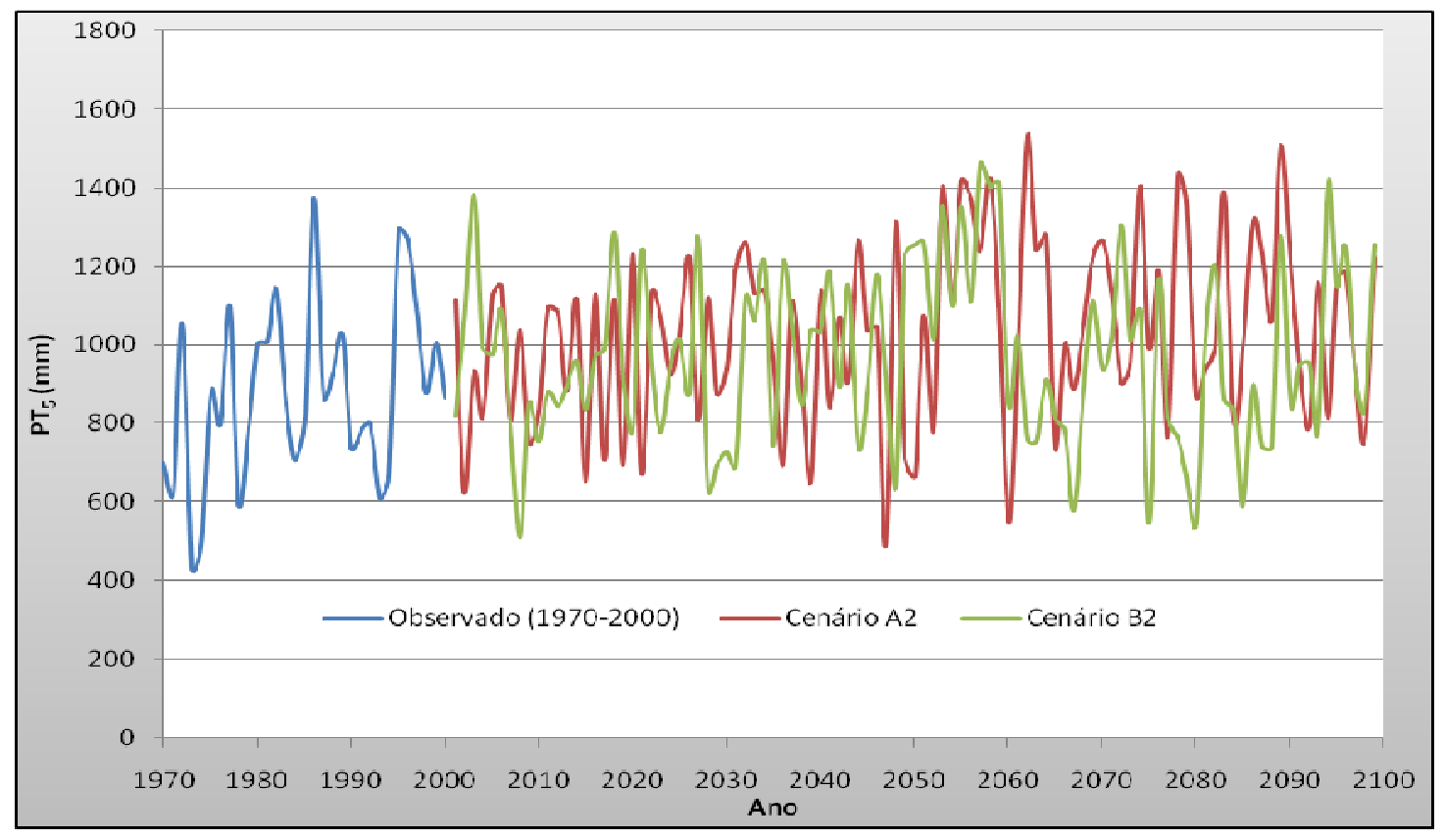

FIGURA 2. Precipitação acumulada nos cinco meses anteriores ao início do período de recessão do escoamento subterrâneo $\left(\mathrm{PT}_{5}\right)$ de 1970 a 2099.Accumulated precipitation from the previous five months $\left(\mathrm{PT}_{5}\right)$ to the beginning of groundwater flow recession period (1970 to 2099).

A partir de 2060, pode-se esperar aumento ainda maior nas precipitações para o cenário A2,

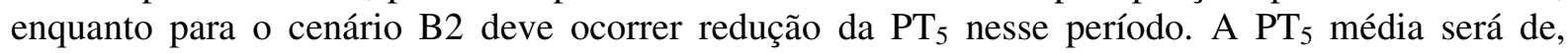
aproximadamente, $1.079 \mathrm{~mm}$ para o cenário A2, e $921 \mathrm{~mm}$ para o cenário B2. 
$\mathrm{Na}$ Tabela 4, são apresentadas as equações de regressão das $\mathrm{q}_{7,10}$ em função do tempo, nos dois cenários, para cada estação fluviométrica. No cenário B2, evidencia-se que, apesar de todas as estações terem apresentado tendência de crescimento nas vazões com o passar do tempo, as equações de regressão não foram significativas.

O comportamento das precipitações ao longo deste século explica os resultados encontrados para as vazões mínimas na bacia do Paracatu, pois a $\mathrm{PT}_{5}$ afeta diretamente o valor da vazão subterrânea no início do período de recessão $\left(\mathrm{Q}_{0}\right)$, como indicado na eq.(2).

TABELA 4. Equações de regressão e $R^{2}$ ajustadas para cada estação fluviométrica. Regression equations and adjusted $R^{2}$ for each flow station.

\begin{tabular}{|c|c|c|c|c|}
\hline \multirow{2}{*}{ Estação } & \multicolumn{2}{|l|}{ Cenário A2 } & \multicolumn{2}{|l|}{ Cenário B2 } \\
\hline & Equação & $\mathrm{r}^{2}$ & Equação & $\mathrm{r}^{2}$ \\
\hline 42250000 & $\mathrm{q}_{7,10}=4,05+0,24 \mathrm{P}$ & 0,48 & $\mathrm{q}_{7,10}=4,08+0,16 \mathrm{P}^{\mathrm{NS}}$ & 0,27 \\
\hline 42251000 & $\mathrm{q}_{7,10}=2,76+0,12 \mathrm{P}$ & 0,51 & $\mathrm{q}_{7,10}=2,77+0,07 \mathrm{P}^{\mathrm{NS}}$ & 0,23 \\
\hline 42255000 & $\mathrm{q}_{7,10}=2,26+0,11 \mathrm{P}$ & 0,51 & $\mathrm{q}_{7,10}=2,29+0,06 \mathrm{P}^{\mathrm{NS}}$ & 0,24 \\
\hline 42257000 & $\mathrm{q}_{7,10}=2,38+0,24 \mathrm{P}$ & 0,50 & $\mathrm{q}_{7,10}=2,41+0,07 \mathrm{P}^{\mathrm{NS}}$ & 0,26 \\
\hline 42290000 & $\mathrm{q}_{7,10}=1,85+0,09 \mathrm{P}$ & 0,58 & $\mathrm{q}_{7,10}=1,80+0,04 \mathrm{P}^{\mathrm{NS}}$ & 0,08 \\
\hline 42395000 & $\mathrm{q}_{7,10}=2,21+0,13 \mathrm{P}$ & 0,57 & $\mathrm{q}_{7,10}=2,05+0,06 \mathrm{P}^{\mathrm{NS}}$ & 0,06 \\
\hline 42435000 & $\mathrm{q}_{7,10}=6,81+0,89 \mathrm{P}$ & 0,53 & $\mathrm{q}_{7,10}=6,21+0,56 \mathrm{P}^{\mathrm{NS}}$ & 0,16 \\
\hline 42440000 & $\mathrm{q}_{7,10}=2,68+0,11 \mathrm{P}$ & 0,49 & $\mathrm{q}_{7,10}=2,69+0,08 \mathrm{P}^{\mathrm{NS}}$ & 0,26 \\
\hline 42460000 & $\mathrm{q}_{7,10}=3,40+0,04 \mathrm{P}^{\mathrm{NS}}$ & 0,18 & $\mathrm{q}_{7,10}=3,42+0,01 \mathrm{P}^{\mathrm{NS}}$ & 0,01 \\
\hline 42490000 & $\mathrm{q}_{7,10}=2,53+0,03 \mathrm{P}^{\mathrm{NS}}$ & 0,19 & $\mathrm{q}_{7,10}=2,54+0,01 \mathrm{P}^{\mathrm{NS}}$ & 0,00 \\
\hline 42540000 & $\mathrm{q}_{7,10}=3,21+0,08 \mathrm{P}^{\mathrm{NS}}$ & 0,24 & $\mathrm{q}_{7,10}=3,22+0,04 \mathrm{P}^{\mathrm{NS}}$ & 0,03 \\
\hline 42545500 & $\mathrm{q}_{7,10}=2,19+0,10 \mathrm{P}$ & 0,36 & $\mathrm{q}_{7,10}=2,12+0,04 \mathrm{P}^{\mathrm{NS}}$ & 0,02 \\
\hline 42546000 & $\mathrm{q}_{7,10}=3,12+0,04 \mathrm{P}^{\mathrm{NS}}$ & 0,11 & $\mathrm{q}_{7,10}=3,03+0,02 \mathrm{P}^{\mathrm{NS}}$ & 0,01 \\
\hline 42600000 & $\mathrm{q}_{7,10}=2,29+0,08 \mathrm{P}$ & 0,43 & $\mathrm{q}_{7,10}=2,12+0,04 \mathrm{P}^{\mathrm{NS}}$ & 0,02 \\
\hline 42690001 & $\mathrm{q}_{7,10}=1,93+0,09 \mathrm{P}$ & 0,51 & $\mathrm{q}_{7,10}=1,86+0,04 \mathrm{P}^{\mathrm{NS}}$ & 0,05 \\
\hline 42750000 & $\mathrm{q}_{7,10}=1,96+0,09 \mathrm{P}$ & 0,52 & $\mathrm{q}_{7,10}=1,88+0,04 \mathrm{P}^{\mathrm{NS}}$ & 0,04 \\
\hline 42840000 & $\mathrm{q}_{7,10}=6,05+0,19 \mathrm{P}$ & 0,41 & $\mathrm{q}_{7,10}=5,86+0,14 \mathrm{P}^{\mathrm{NS}}$ & 0,19 \\
\hline 42850000 & $\mathrm{q}_{7,10}=1,55+0,12 \mathrm{P}$ & 0,52 & $\mathrm{q}_{7,10}=1,38+0,06 \mathrm{P}^{\mathrm{NS}}$ & 0,05 \\
\hline 42860000 & $\mathrm{q}_{7,10}=1,27+0,07 \mathrm{P}$ & 0,51 & $\mathrm{q}_{7,10}=1,17+0,04 \mathrm{P}^{\mathrm{NS}}$ & 0,07 \\
\hline 42930000 & $\mathrm{q}_{7,10}=1,93+0,09 \mathrm{P}$ & 0,52 & $\mathrm{q}_{7,10}=1,84+0,04 \mathrm{P}^{\mathrm{NS}}$ & 0,06 \\
\hline 42980000 & $\mathrm{q}_{7,10}=2,03+0,12 \mathrm{P}$ & 0,53 & $\mathrm{q}_{7,10}=1,92+0,06 \mathrm{P}^{\mathrm{NS}}$ & 0,07 \\
\hline
\end{tabular}

$\mathrm{P}$ - período considerado, sendo $\mathrm{P}=0$ corresponde ao período de 1970-2000; $\mathrm{P}=1$ corresponde ao período de 2001$2010, \ldots, P=10$ corresponde ao período de 2090-2099; ${ }^{\text {NS }}$ - regressão não-significativa $(P>0,05)$.

De acordo com a Tabela 4, para 17 das 21 estações estudadas, as equações de regressão são significativas, quando se considera o cenário A2. Nesse cenário, fica evidenciada tendência de aumento nas vazões mínimas com o passar do tempo, em todas as estações estudadas, devido à tendência de aumento da $\mathrm{PT}_{5}$ em toda a bacia a partir de 2060, como discutido anteriormente. Por outro lado, no cenário B2, há tendência de redução na $\mathrm{PT}_{5}$ a partir de 2060 , que, por sua vez, pode levar à redução nas vazões mínimas.

Na Figura 3, é apresentado o exemplo correspondente à estação 42290000, nos cenários A2 e B2. Destaca-se o fato de que o comportamento apresentado por essa estação é representativo do comportamento observado nas outras estações utilizadas neste trabalho. Na figura, pode-se evidenciar a maior dispersão dos pontos no cenário B2, o que explica, em parte, os baixos valores de $r^{2}$ e a ausência de significância das equações de regressão nesse cenário.

Com base nas equações de regressão obtidas no cenário A2, na Tabela 5, apresentam-se os acréscimos esperados na $\mathrm{q}_{7,10}$ para o período de 2090-2099, em relação ao período correspondente a 1970-2000. Como nenhuma das equações de regressão obtidas no cenário B2 foram significativas, os acréscimos esperados para a $\mathrm{q}_{7,10}$, nesse cenário, foram omitidos. 


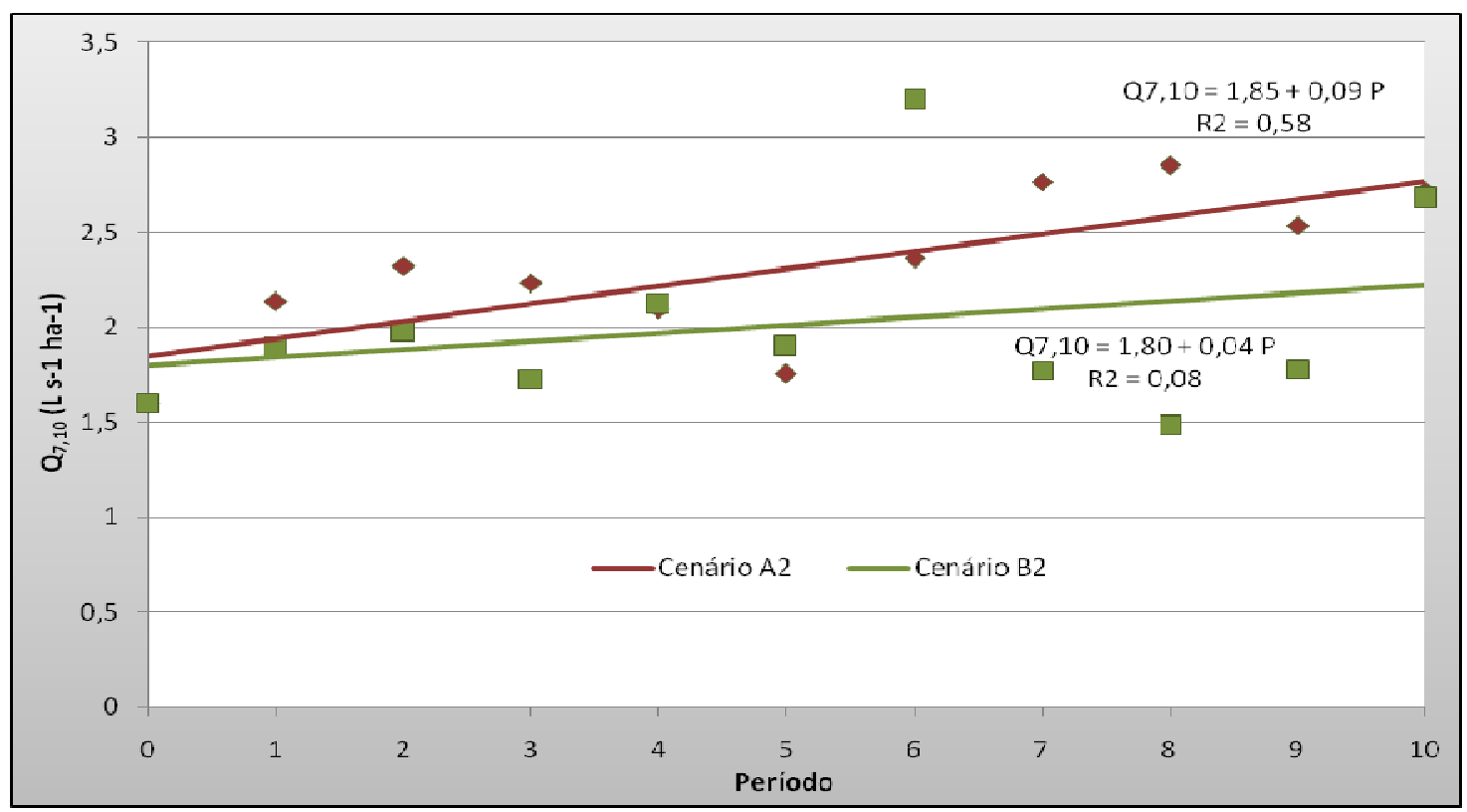

FIGURA 3. Q ${ }_{7,10}$ específica na estação 42290000 (BR-040 - Paracatu). Specific $\mathbf{q}_{7,10}$ in the 42290000 flow station (BR-040 - Paracatu).

TABELA 5. Q Q $_{7,10}$ específicas estimadas pelas equações de regressão, para os períodos de 19702000 e 2090-2099, e acréscimo esperado nesta variável no período considerado para o cenário A2. Specific $\mathbf{q}_{7,10}$ estimated with the regression equations for the 19702000 and 2090-2099 periods, and expected increase at the last period in the scenario A2.

\begin{tabular}{cccc}
\hline \multirow{2}{*}{ Estação } & $1970-2000$ & \multicolumn{2}{c}{$2090-2099$} \\
\cline { 2 - 4 } & $\mathrm{q}_{7,10}\left(\mathrm{~L} \mathrm{~s}^{-1} \mathrm{ha}^{-1}\right)$ & $\mathrm{q}_{7,10}\left(\mathrm{~L} \mathrm{~s}^{-1} \mathrm{ha}^{-1}\right)$ & Acréscimo (\%) \\
42250000 & 4,1 & 6,5 & 59,0 \\
42251000 & 2,8 & 4,0 & 43,0 \\
42255000 & 2,3 & 3,4 & 48,0 \\
42257000 & 2,4 & 4,8 & 100,0 \\
42290000 & 1,9 & 2,8 & 47,0 \\
42395000 & 2,2 & 3,5 & 59,0 \\
42435000 & 6,8 & 15,7 & 131,0 \\
42440000 & 2,7 & 3,8 & 41,0 \\
42460000 & 3,4 & 3,8 & 12,0 \\
42490000 & 2,5 & 2,8 & 12,0 \\
42540000 & 3,2 & 4,0 & 25,0 \\
42545500 & 2,2 & 3,2 & 45,0 \\
42546000 & 3,1 & 3,5 & 13,0 \\
42600000 & 2,3 & 3,1 & 35,0 \\
42690001 & 1,9 & 2,8 & 47,0 \\
42750000 & 2,0 & 2,9 & 45,0 \\
42840000 & 6,1 & 8,0 & 31,0 \\
42850000 & 1,6 & 2,8 & 75,0 \\
42860000 & 1,3 & 2,0 & 54,0 \\
42930000 & 1,9 & 2,8 & 47,0 \\
42980000 & 2,0 & 3,2 & 60,0 \\
NS - estações para as quais as equações de regressão não foram significativas, como apresentadas na Tabela 4.
\end{tabular}


$\mathrm{Na}$ Tabela 5, pode-se evidenciar que os acréscimos nas $\mathrm{q}_{7,10}$, para o final deste século, variam de 31 a 131\%. Embora os acréscimos esperados nas estações 42460000; 42490000; 42546000 e 42540000 sejam de $12 ; 12 ; 13$ e $25 \%$, respectivamente, as equações de regressão para essas estações não foram significativas, como já apresentadas na Tabela 4. Essas quatro estações localizam-se no trecho norte da bacia. Por outro lado, as estações 42980000; 42850000; 42257000 e 42435000, que apresentaram maior tendência de acréscimo na $\mathrm{q}_{7,10}$, não estão concentradas em uma única região, ao contrário, localizam-se em diferentes pontos da bacia, assim como as demais estações que apresentaram tendência de acréscimo intermediário.

\section{CONCLUSÕES}

Para o Cenário A2 (maior emissão de gases de efeito estufa), há tendência significativa de aumento da disponibilidade hídrica $\left(\mathrm{q}_{7,10}\right)$ em $81 \%$ das estações fluviométricas da bacia do Rio Paracatu que foram analisadas neste trabalho. $\mathrm{O}$ aumento da disponibilidade hídrica $\left(\mathrm{q}_{7,10}\right)$, nas diferentes estações fluviométricas, variou de 31 a $131 \%$ até o final deste século.

Para o Cenário B2 (menor emissão de gases de efeito estufa), as tendências de aumento da disponibilidade hídrica $\left(\mathrm{q}_{7,10}\right)$ até o final deste século não foram significativas para nenhuma das estações fluviométricas utilizadas neste trabalho.

\section{REFERÊNCIAS}

HULME, M.; SHEARD, N. Cenários de alterações climáticas para o Brasil. Norwich: Climate Research Unit, 1999. 6 p. Disponível em: <www.cru.uea.ac.uk/ mikeh/research/brazil.pdf>. Acesso em: 6 nov. 2006

IPCC. Climate change 2001: Impacts, adaptation, and vulnerability. In: MCCARTHY, J.J. Contribution of Working Group II to the Third Assessment Report of the Intergovernmental Panel on Climate Change. Cambridge: Cambridge University Press, 2001. p.1.032.

IPCC. Climate change 2007: The Physical Science Basis. In: SOLOMON, S.D.; QIN, M.; MANNING, Z.; CHEN, M.; MARQUIS, K.B.; AVERYT, M.T.; MILLER, H.L. (Eds.). Contribution of Working Group I to the Fourth Assessment Report of the Intergovernmental Panel on Climate Change. Cambridge: Cambridge University Press, 2007. p.996.

IPCC-TGCIA. Guidelines on the Use of Scenario Data for Climate Impact and Adaptation Assessment. Version 1. In: CARTER, T.R.; HULME, M.; LAL, M. Intergovernmental Panel on Climate Change, Task Group on Scenarios for Climate Impact Assessment, 1999. p.69.

KROL, M.S.; BRONSTERT, A. Regional integrated modelling of climate change impacts on natural resources and resource usage in semi-arid Northeast Brazil. Environmental Modelling \& Software, Oxford, v.22, p.259-68, 2007.

KROL, M.; JAEGER, A.; BRONSTERT, A.; GÜNTNER, A. Integrated modelling of climate, water, soil, agricultural and socio-economic processes: A general introduction of the methodology and some exemplary results from the semi-arid north-east of Brazil. Journal of Hydrology, Amsterdam, v.328, p.417-31, 2006.

NOBRE, C.A. Mudanças climáticas globais: possíveis impactos nos ecossistemas do País. Parcerias Estratégicas, Brasília, n.12, p.239-58, 2001.

NOVAES, L. F. Modelo para a quantificação da disponibilidade hídrica na bacia do Paracatu. 2005. 104 f. Dissertação (Mestrado em Recursos Hídricos) - Universidade Federal de Viçosa, Viçosa - MG, 2005.

RODRIGUEZ, R.D.G. Metodologia para estimativa das demandas e disponibilidades hídricas: estudo de caso da bacia do Paracatu. 2004. 94 f. Dissertação (Mestrado em Recursos Hídricos) Universidade Federal de Viçosa, Viçosa - MG, 2004.

ROSENZWEIG, C.; STRZEPEKB, K.M.; DAVID, C.; MAJORC, D.C.; IGLESIAS, A.; YATES, D.N.; McCLUSKEY, A.; HILLEL, D. Water resources for agriculture in a changing climate: international case studies. Global Environmental Change, Guildford, v.14, p.345-60, 2004. 